\title{
ACCURACY ASSESSMENT OF MOBILE MAPPING POINT CLOUDS USING THE EXISTING ENVIRONMENT AS TERRESTRIAL REFERENCE
}

\author{
S. Hofmann ${ }^{\text {a }}$, C. Brenner ${ }^{\mathrm{a}}$ \\ ${ }^{a}$ Institute of Cartography and Geoinformatics, Leibniz Universität Hannover, Germany - \\ \{sabine.hofmann, claus.brenner\}@ikg.uni-hannover.de
}

Commission V, ICWG I/V-A

KEY WORDS: Accuracy Assessment, Mobile Mapping, Point Cloud, Reference Data, Total Station, 3D Test Field, Facades, Street profile

\begin{abstract}
:
Mobile mapping data is widely used in various applications, what makes it especially important for data users to get a statistically verified quality statement on the geometric accuracy of the acquired point clouds or its processed products. The accuracy of point clouds can be divided into an absolute and a relative quality, where the absolute quality describes the position of the point cloud in a world coordinate system such as WGS84 or UTM, whereas the relative accuracy describes the accuracy within the point cloud itself. Furthermore, the quality of processed products such as segmented features depends on the global accuracy of the point cloud but mainly on the quality of the processing steps. Several data sources with different characteristics and quality can be thought of as potential reference data, such as cadastral maps, orthophoto, artificial control objects or terrestrial surveys using a total station.

In this work a test field in a selected residential area was acquired as reference data in a terrestrial survey using a total station. In order to reach high accuracy the stationing of the total station was based on a newly made geodetic network with a local accuracy of less than $3 \mathrm{~mm}$. The global position of the network was determined using a long time GNSS survey reaching an accuracy of $8 \mathrm{~mm}$. Based on this geodetic network a 3D test field with facades and street profiles was measured with a total station, each point with a two-dimensional position and altitude. In addition, the surface of poles of street lights, traffic signs and trees was acquired using the scanning mode of the total station.

Comparing this reference data to the acquired mobile mapping point clouds of several measurement campaigns a detailed quality statement on the accuracy of the point cloud data is made. Additionally, the advantages and disadvantages of the described reference data source concerning availability, cost, accuracy and applicability are discussed.
\end{abstract}

\section{INTRODUCTION}

Dense point clouds and a fast data capturing technology made mobile mapping a widely used method for data acquisition. Various applications such as mapping and map updates or 3D city modelling, e.g. for planning, require a verified quality statement on the geometric accuracy of the acquired point clouds or image data.

A quality statement about the acquired mobile mapping data can be made by using reference data sources, such as cadastral maps, orthophotos, artificial test fields, or data from terrestrial surveys of the environment. Several examples of accuracy analysis and validation of Mobile Mapping Systems can be found in the literature, e.g. (Graefe et al., 2001), (Kremer and Hunter, 2007), (Haala et al., 2008), (Puente et al., 2012) and (Toschi et al., 2015), which will be discussed in the following paragraphs.

\subsection{Cadastral Data as Reference}

Cadastral maps have the advantage, that they are widely available and contain geometric information about buildings, sometimes 3D building models, roads, sidewalks, and commonly further objects such as traffic lights or trees, which can be used for comparison with features extracted from mobile mapping point clouds. (Haala et al., 2008) use 3D city models for analysing the accuracy of a mobile mapping point cloud.
A drawback might be the generalization during the digitalisation process due to complex building structures or their actuality. The accuracy of the cadastral data is usually in the range of a few centimetres to decimetres and therefore in some cases unsatisfactory.

The use of orthophotos mainly shows the problem of different data capturing directions and thus leads to different shaping and visibility of objects, which results in problems during the assignment of identical objects. In case of buildings, orthophotos only show the roof tops in contrast to the facades visible in the mobile mapping data.

\subsection{Artificial Test Fields as Reference}

Artificial test fields can be either signalled target objects distributed in the environment or specifically designed portable calibration objects. An accuracy analysis using a test field with artificial control points is presented in (Graefe et al., 2001), who assess the accuracy of the trajectory data of a mobile mapping system using control points captured with a stereo camera system. The control points reach an accuracy of about $10 \mathrm{~cm}$ in position and $5 \mathrm{~cm}$ in height. The 46 control points where distributed along a $5 \mathrm{~km}$ route under representative Central European GNSS conditions.

The use of a specifically designed calibration object is discussed in (Puente et al., 2012). The $3 \mathrm{mx} 3 \mathrm{~m}$ test field consists of 5 spheres with $100 \mathrm{~mm}$ in diameter precisely

* Corresponding author 
calibrated. This work shows a relative accuracy of the mobile mapping system of about $10 \mathrm{~mm}$, with no correlation between the accuracy and the distance to the test field. The absolute accuracy depending on GNSS conditions was in the range of a few centimetres to $30 \mathrm{~cm}$.

\subsection{Terrestrial Survey of the Environment}

Without the use of special target objects, a terrestrial survey can be used for a detailed analysis of mobile mapping data in a limited area. An extensive analysis of the precision and accuracy of mobile mapping point clouds show (Kremer and Hunter, 2007). Reference control points on the road surface were acquired using RTK satellite navigation. Height differences were determined using points on the road surface, accuracy in position was determined using corner points on street markings. The quality of the results is limited by the accuracy of the control points which is in the range of decimetres using RTK. (Toschi et al., 2015) describe the use of reference point clouds from a photogrammetric survey as well as from a terrestrial laser scanner. They present an advanced statistical approach analysing the quality of a mobile mapping point cloud.

Using the existing environment, such as buildings, poles or the road surface has several advantages. In the first place, no artificial targets have to be installed and maintained. In comparison to cadastral data, a terrestrial survey provides features which might not be included in cadastral maps, such as height information or most pole-like objects. Furthermore, in a terrestrial survey, points are acquired on the "surface" of buildings which can be different to cadastral data where the insulation or plaster of a facade is not considered. Since the insulation can be up to several decimetres, this is a significant source of errors. Additionally, a terrestrial survey based on a geodetic network provides detailed information about the accuracy of each reference point within the test field.

\section{REFERENCE DATA}

In this work, reference data was acquired in a terrestrial survey in a selected residential area, which has a variety of different building types, such as detached houses and 4-storey apartment buildings, as well as different GNSS conditions caused by trees or wide and narrow streets. Furthermore, a large set of mobile mapping data was acquired within this area over a longer period of time which can be used for further accuracy analysis over time.

The data acquisition for the reference data is described in detail in the following paragraphs. In order to reach a high local and global accuracy for each reference point, all measurements are based on a geodetic network with the global position determined using static GNSS measurements. As reference objects for the horizontal positions, selected planar objects, such as vertical facades, were captured with single point measurements. In order to determine a height reference, street profiles were captured in addition.

\subsection{Local Reference by a Geodetic Network}

The local reference for the planned test field is based on a geodetic network with 17 points in total. All network points had to be carefully chosen since there was no line of sight between points on opposite corners of the network. Therefore, points along each road axis where chosen on opposite sides of the roads in order to get a higher stability within the network. As further condition, points not located on intersections should be visible from at least three other points. Due to occlusions from parked vehicles, points 112 and 510 do not satisfy this condition. The network points were marked stably with steel measuring pins on sidewalks.

For the measurements the Leica TS 30 total station (Leica, 2016) was used with a distance measurement accuracy of $0.6 \mathrm{~mm}+1 \mathrm{ppm}$ and an angular measurement accuracy of 0.1 mgon. For high accuracies we used tripods with standard prisms to signal all network points with forced centring. All measurements were carried out in one day with a final measurement as loop closure and control to the start point of the measurements.

The adjustment of the measured network was carried out as free network adjustment with total track minimization using standard adjustment software. The resulting standard deviation for the $2 \mathrm{D}$ position is between $0.4 \mathrm{~mm}$ and $1.1 \mathrm{~mm}$ with a mean value of $0.5 \mathrm{~mm}$. The height of the points is given with a standard deviation between $0.6 \mathrm{~mm}$ and $1.3 \mathrm{~mm}$. The standard deviation of the position is shown for each network point as green ellipses in Fig. 1. Due to the better geometry of the measurement angles, the points on cross roads reach a better accuracy, which is shown by smaller circular error ellipses, e.g. at point 200 and 450 (Fig. 1), than along roads, e.g. points 350 or 480 .

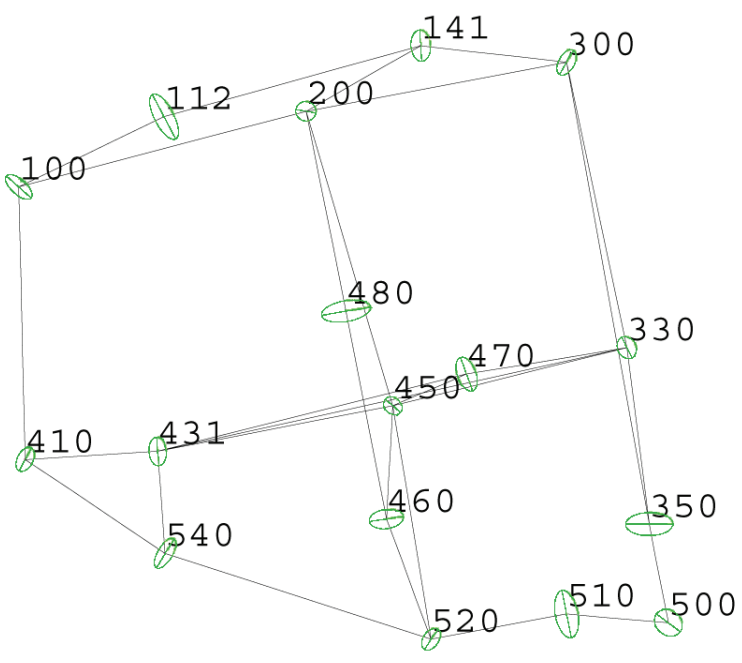

Figure 1. Geodetic network for the test area of $300 \mathrm{~m} \times 300 \mathrm{~m}$ : GNSS measurements on points 100, 300 and 500, green ellipses show (scaled) standard deviation of points. The largest error occurs at point 480 , where the main axis of the ellipse has a length of $1.1 \mathrm{~mm}$.

\subsection{Global Reference using GNSS}

The global reference within the test area was determined using static GNSS measurements on three selected points of the network. In order to reach a high stability of the network, these three points were selected at the edges of the area (Fig. 1, points 100, 300 and 500). Each point was observed twice, each observation with a duration of $2 \mathrm{~h}$. In order to work with forced centring during the geodetic network measurements a special clamp was used installing a prism in conjunction with the GNSS antenna.

For the measurements a Leica System 1200 with a Leica ATX1230 SmartAntenna and a RX 1210 receiver was used in static mode, collecting raw data with a measurement rate of 
1 second. Additionally, precise real time reference data from an AXIO-NET reference station was received during the measurement epoch (AXIO-NET, 2016). Furthermore, for post processing data from a local SAPOS reference station was used with a data rate of 1 second (LGLN, 2016).

Post processing was carried out using the Software Leica GeoOffice. An elevation angle of $20^{\circ}$ was selected since the surrounding of the points contains a few obstacles at lower angles. GPS only was used as GNSS type with L1 frequency and precise ephemerides with fixed phase solution. As results the coordinates of the three observed points where determined with an accuracy of $6.6 \mathrm{~mm}(1 \sigma)$.

\subsection{Test Field in Urban Environment}

In this work existing anthropogenic structures in urban environments without special signalling or targets are used as reference objects. The main requirements were the visibility and recognisability of the objects in the mobile mapping point clouds. Therefore, all measured points had to be visible from public drivable roads. Furthermore, the objects along each road should cover diverse orientations for optimal accuracy analysis of the mobile mapping data. The selected test area is shown in Fig. 2 in an orthophoto overlaid by cadastral building footprints and road boundaries. Red dots show the reference data on facades. Since the data has not been acquired at the same time, differences may occur. Centred in the bottom half of the image one building was torn down and a new building was constructed.

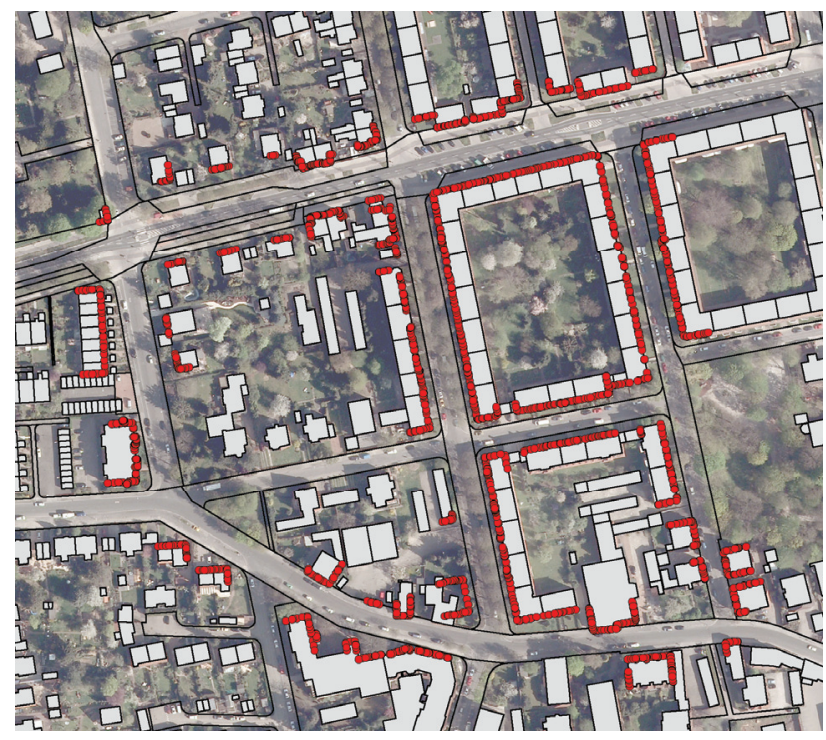

Figure 2. Test area in Hannover, Germany, with an area of $300 \mathrm{~m}$ x $300 \mathrm{~m}$. Orthophoto overlaid with building footprints (gray area), road boundaries (black lines) and measured facade points (red dots).

The reference data was captured with the Leica Nova MS 50 MultiStation (Leica, 2016), which has an additional scan mode for capturing 3D information of a user defined area or object. Additional image information can be acquired using the integrated coaxial camera with 5 MPixel CMOS sensor. The accuracy of the angle measurements is given by the manufacturer with $0.3 \mathrm{mgon}$, the distance measurement accuracy is in the range of $2 \mathrm{~mm}+2 \mathrm{ppm}$ for non-prism measurements on any surface. The non-prism measurement mode has a maximum range of $2000 \mathrm{~m}$, but in the case of facade measurements in the defined test field the maximum ranges where about $20 \mathrm{~m}-30 \mathrm{~m}$. During the measurements the instruments position is determined using free stationing based on at least three points of the geodetic network, where the instrument determines the quality of each stationing directly after calculating its position.

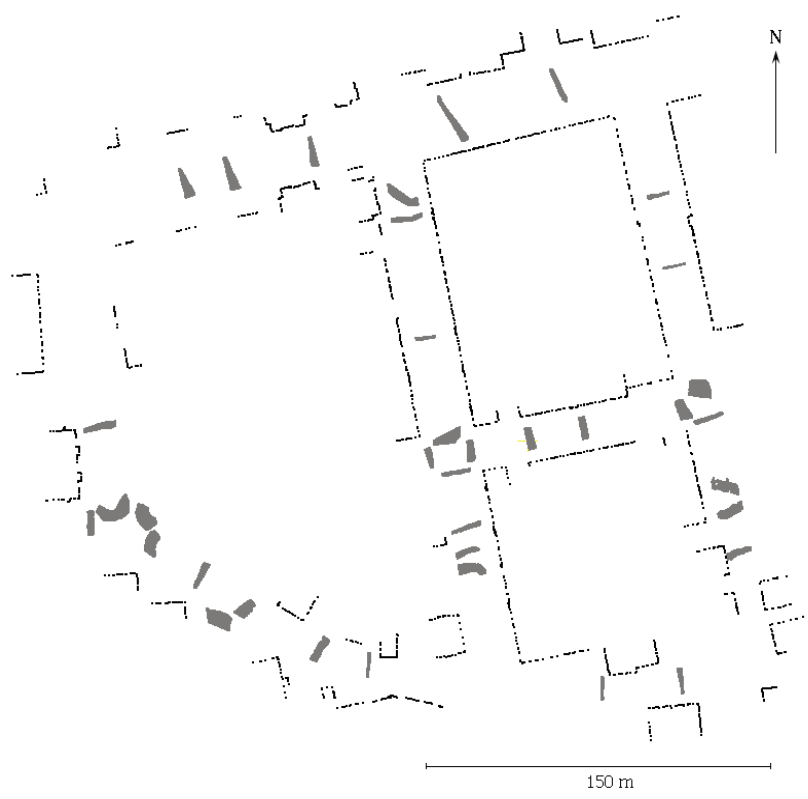

Figure 3. 3D test field with measured points on facades (black) and street profile measurements (gray).

The test field was acquired within four days, were we measured about 4000 points on facades covering each facade plane with up to 20 single points (Fig. 3). Additionally, small planar elements on the facade such as ledges or doorways were covered with points. Each point has a unique ID by which they can be identified as belonging to the same plane. All points can be used as single points for comparison with mobile mapping data. Furthermore, using all points belonging to one plane, the parameters can be determined for modelling planar objects.

In addition, georeferenced street profiles were captured using the scanning mode of the Leica MS 50 since their surface cannot be described with a few points (Fig. 3, gray areas). Street profiles are used for height reference of the mobile mapping data, since the facades provide mainly position but not height information. In the test field a total of 37 street profiles transverse to the road axis with a width of $1 \mathrm{~m}$ and a minimal resolution of $5 \mathrm{~cm}$ in $\mathrm{x}-\mathrm{y}$-plane were measured.

\section{MOBILE MAPPING}

\subsection{Mobile Mapping System VMX-250}

Mobile mapping data was acquired using the RIEGL VMX-250 mobile mapping system (Riegl, 2012) equipped with two calibrated laser scanners, four cameras and the precise GNSSIMU-System POS-LV 510 (Applanix, 2016) for positioning and orientation.

The laser scanners have a maximum data rate of 600,000 points per second in total, captured with $360^{\circ}$ profile measurements at 100 profiles, so called scan lines, per second. Therefore, the density of the point cloud along track is mainly influenced by the vehicle speed and can be estimated with $1 \mathrm{~cm}$ per $\mathrm{m} / \mathrm{s}$. Across track, the spacing of the points is $2 \mathrm{~mm}$ per meter distance, which is usually more dense. The driving speed during 
data capturing is normally between $30 \mathrm{~km} / \mathrm{h}$ and $80 \mathrm{~km} / \mathrm{h}$ depending on the speed limit and the traffic.

The measurement accuracy of the laser scanners is specified by the manufacturer with $10 \mathrm{~mm}$ accuracy and $5 \mathrm{~mm}$ precision (both $1 \sigma$ ). The absolute accuracy of the point cloud, which is analyzed in this work, mainly depends on the positioning and orientation performance of the GNSS-IMU-System, especially GNSS outages lead to a loss in accuracy of about $15-30 \mathrm{~cm}$. The relative accuracy in a local area, e.g. along a building or street section, which is also subject of this work, depends on the IMU and the scanner calibration relative to the GNSS-IMUsystem and is usually in the range of a few centimetres. The scanners are fixedly mounted on the GNSS-IMU-System and manufacturer calibrated.

\subsection{Mobile Mapping Point Clouds}

The point cloud data from the RIEGL VMX-250 can be assessed in two different modes: scan strips and 3D point clouds. Scan strips contain the acquired points as one strip along the trajectory, where points on consecutive scan lines are sorted in consecutive columns, which therefore also contain the local neighbourhood of each point. Neighbouring points along each scan line are stored in adjacent rows, which means in case of the used scanners, that neighbouring pixels in the strip were captured with a time difference of $10 \mathrm{~ms}$ or $3 \mu \mathrm{s}$, for horizontal and vertical neighbours, respectively.

The main advantage of this representation is the preserved measurement adjacency relationship, which is lost in a Cartesian 3D point cloud representation. Furthermore, as the strips are rectangular arrays, they can be worked on and displayed as images were image processing algorithms can be used. Since the 3D point coordinates are stored in those arrays, no information of the raw data is lost. Furthermore, additional information such as reflectance or timestamp of each point can be stored, the latter being important to relate each scanned point to the location of the mapping van at the time it was captured. Based on the scan strips, a local normal vector is computed for each single point. A robust estimation of a local plane is used for each point so that no blurring occurs at discontinuities, such as height jumps or crease edges (e.g. building corners).

\section{COMPARISON OF MOBILE MAPPING POINT CLOUDS AND REFERENCE DATA}

In this work, the accuracy is differentiated into absolute and relative accuracy. The absolute accuracy describes the accuracy of the captured data in a world coordinate system such as WGS84 or projected in UTM, the relative accuracy gives a statement about the geometric accuracy within the point cloud itself, e.g. the correct distance between facades on opposite sides of a street.

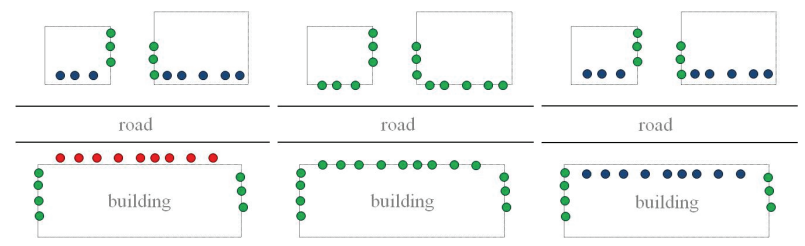

Figure 4. Illustration of absolute and relative accuracy: absolute accuracy low across street (left), absolute and relative accuracy high (middle), relative accuracy low (right).

\subsection{Distinction of Absolute and Relative Accuracy}

4.1.1 Absolute Accuracy: the absolute accuracy shows the positioning errors of the mobile mapping system based on the GNSS-IMU system, which directly leads to errors in the global position of the entire point cloud (Fig. 4, left, middle).

Due to "local" effects caused by multi path and occlusion of the GNSS signal, the absolute accuracy within any single point cloud may change. These local differences in accuracy are still visible after a rigid transformation of the mobile mapping point cloud to control points from reference data. In addition to multi path or occlusions of the GNSS signal, the effect of different transformation parameters between global coordinate systems such as WGS84 and ETRS89 has to be taken into account when comparing point clouds to a global reference data set. Especially the use of geoid information for transformations between height systems can cause an additional offset in the point cloud data. In this work, this effect is not analyzed separately.

The absolute accuracy of mobile mapping point clouds is especially relevant for comparing or integrating the mobile mapping point clouds with other data sources, e.g. for map updates or modelling buildings for 3D city models. Additionally, for matching point clouds from different epochs the absolute accuracy of the point clouds is important as prior knowledge for automatic matching algorithms.

4.1.2 Relative Accuracy: the relative accuracy describes the accuracy within the mobile mapping point cloud for regions of spatially limited extent. Such regions may be short street corridors or intersections, where the absolute accuracy is stable and mainly depends on the IMU system and the scanner technology. That means the relative accuracy is determined based on distances between neighbouring objects within the point cloud, such as buildings on opposite sides of a road. Even with a low global accuracy, the relative accuracy can be determined using reference data on opposite facades. For example, in case of a global shift of the scan data, points on opposite facades may be in front of the reference on one side of the road and behind the reference on the opposite side. Applying the correct shift between point cloud and reference, this effect should decrease and corresponding points from reference and mobile mapping data should coincide (Fig. 4. middle, right).

The relative accuracy can be used for a GNSS independent quality analysis of mobile mapping data, e.g. in maps for autonomous driving, a high local accuracy is much more relevant than a high global accuracy for the positioning of any vehicle.

\subsection{Accuracy Evaluation based on Trajectory Adjustment}

4.2.1 Deviation between reference and point cloud data: the deviation between reference data and any point cloud is determined by computing, for every point in the reference data, the orthogonal distance to the closest surface in the point cloud. The closest surface is obtained by finding the closest scan point in Euclidean space. The distance is then computed based on the local plane of this point (estimated as described in section 3.2). Using this point-to-plane distance is more accurate than using the point-to-point distance. Also, since the local normal vector is oriented, signed distances are obtained, which allows to distinguish between points in front of and behind the surface. 
4.2.2 Global least squares adjustment: in order to align the point clouds to the reference data, a least squares adjustment was performed. Since orientations are usually very accurate (as opposed to positions), only the translational component was estimated. Each correspondence between a reference point and a surface point leads to a single (dot product) equation:

$$
0+v=\left\langle n_{i}, p_{\text {ref }}-\left(p_{i}+t\right)\right\rangle
$$

where $v$ is the residual, $n_{i}$ is the local normal vector, $p_{\text {ref }}$ is the (fixed) reference point, $p_{i}$ is the surface point (of the laser scan) and $t$ is the unknown global 3D shift vector to be estimated. Note that we do not use point-to-point equations here (which would result in three equations per point correspondence), as this would introduce additional constraints perpendicular to the normal vector. For the global estimation of the translation component $t$, correspondences between each reference point and each point in each scan strip are considered. To yield a robust result, a standard iterative closest point (ICP) approach is used, where the maximum allowed (absolute) distance is $30 \mathrm{~cm}$ initially, which is reduced during the iterations to a final value of $5 \mathrm{~cm}$. Note that equation (1) is linear, so that the need for an iterative adjustment arises only due to the recalculation of point correspondences.

4.2.3 Strip adjustment: Since a single global estimation is not sufficient, we implemented a strip adjustment procedure. Instead of estimating a single, global translation parameter $t$, the translation vector now varies along each scan trajectory. To model the translation along a trajectory, we define "anchor points", which are linearly spaced along the trajectory (at fixed distances). For each anchor point, a translation $t_{j}$ is estimated. Each point correspondence (between a reference point and a surface point) occurs at a specific location along the mobile mapping van trajectory, which can be identified using the time stamp. This location will be between two anchor points, say, $j$ and $j+1$, and consequently, this will lead to observation equations for $t_{j}$ and $t_{j+1}$. The type of equations is identical to equation (1). Since the VMX-250 system runs two scanners simultaneously, the trajectory will be adjusted by observations from both strips. The assignment of observations to corresponding anchor points is illustrated in Fig. 5.

In contrast to the global estimation of $t$, where we can assume that the overall scene contains normal vectors which span 3D space, the local estimation of the anchor points $t_{j}$ will sometimes lead to singular cases. For example, if all observations assigned to a specific anchor point $t_{j}$ involve points on the road surface, where normal vectors point upwards, the estimation of $t_{j}$ will lead to an estimation of the height component of $t_{j}$, but will leave the position (the other two components) undetermined. We decided to remove these singularities by adding weak constraints, through the addition of observation equations ("observed unknowns") of the type:

$$
0+v=t_{j}
$$

for each anchor point $j$. This is justified by the fact that the original trajectories were observed using the GPS-IMU system. Thus, equations (2) can be assumed to hold, with an appropriate weight derived from the characteristics of the GPS-IMU system, as given in the datasheet. However, as we show later, we finally opted to set this weight very low, so that its purpose is solely to remove the degeneracy of the normal equation system.
Finally, it can be assumed that the required adjustments along a trajectory will be smooth, since the mapping system is relying on an IMU system. Thus, the variations in a local neighbourhood should be bounded. We model this by using simple constraint equations between anchor points, of the form:

$$
0+v=t_{j}-t_{j-1}
$$

which are instantiated for any successive anchor points for which observation equations were found. By varying the weight of these equations, the stiffness of the estimated correction along the trajectory can be controlled. In general, this weight should be chosen according to the quality of the used IMU. Since equation(3) uses equally spaced anchor points, it introduces "stiffness in space". One could also think of an alternative approach that uses "stiffness in time" by setting up similar equations for anchor points with equally spaced time stamps. Using equations (1) - (3), the translations of all anchor points are estimated simultaneously. As in the global case, the estimation iterates point assignment and parameter adjustment, starting from a maximum distance of $30 \mathrm{~cm}$ which is narrowed down to $5 \mathrm{~cm}$ in the final iterations.

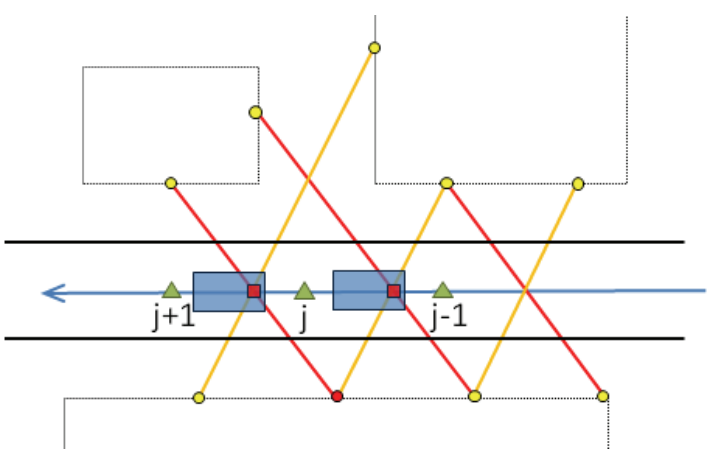

Figure 5. Influence of one object (red dot) on three anchor points $(\mathrm{j}-1, \mathrm{j}, \mathrm{j}+1$, green triangles) on trajectory (blue arrow) with two vehicle positions (blue rectangles). The object is visible from scanner 1 (red line) and has influence on translation $t_{j}$ and $t_{j+l}$ and from scanner 2 (orange line) with influence on $t_{j-1}$ and $t_{j}$.

\section{RESULTS AND DISCUSSION}

\subsection{Data Set}

In this work data from 56 mobile mapping point clouds acquired at 28 epochs with the RIEGL VMX-250 system were used for analysis of position and height accuracy. The different epochs were acquired in 2012 (12 epochs) and 2013 (16 epochs). The point clouds for each epoch are available separately for scanner 1 and 2 .

The used reference data set was acquired in 2015, which might lead to differences in data e.g. caused by construction. From the reference data set 9180 points were used, 4019 on facades and 5161 points from street profiles.

\subsection{Results}

In the following section, all results are shown exemplarily for one selected epoch. At the end of this section, the results for all 28 evaluated epochs are presented.

The selected scan was acquired along a $130 \mathrm{~m}$ long trajectory, with a total of 1392 (1493) reference points visible in scanner 1 (2), from which 1365 (1470) reference points were used in the 
final adjustment. The colour coded deviation between reference data and point clouds before the adjustment of the trajectory are shown for the selected epoch in Fig. 6. The colour coding shows deviations from $-5 \mathrm{~cm}$ in red to $+5 \mathrm{~cm}$ in blue, with $0 \mathrm{~cm}$ in green. Negative deviations imply that the reference point is behind the plane of the mobile mapping point cloud, positive imply that it is in front.
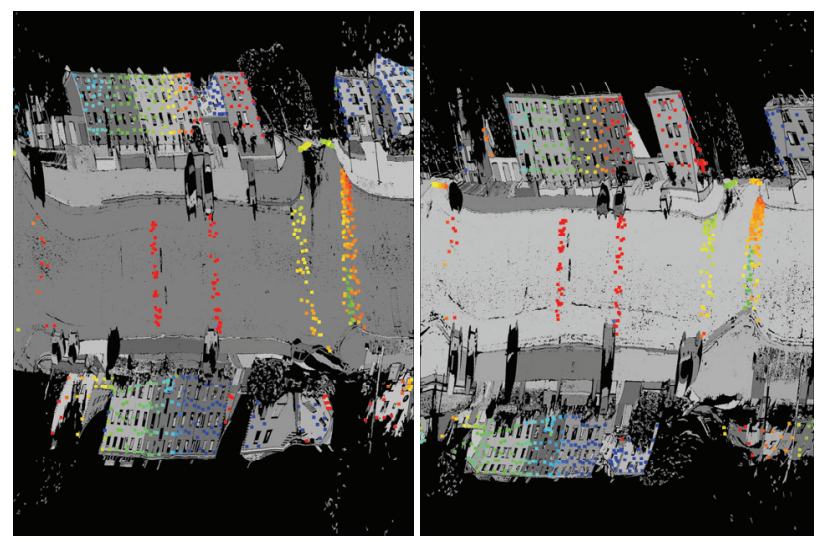

Figure 6. Colour coded deviations between reference and point cloud before adjustment of the trajectory for scanner 1 (left) and scanner 2 (right) with red $=+5 \mathrm{~cm}$, green $=0 \mathrm{~cm}$, blue $=-5 \mathrm{~cm}$.

Histograms of the determined deviations are presented in Fig. 7 for scanner 1 and 2, with a mean value for scanner 1 (2) of $1.1 \mathrm{~cm}(1.8 \mathrm{~cm})$ and standard deviation of $4.2 \mathrm{~cm}(4.0 \mathrm{~cm})$, which indicates an overall shift.

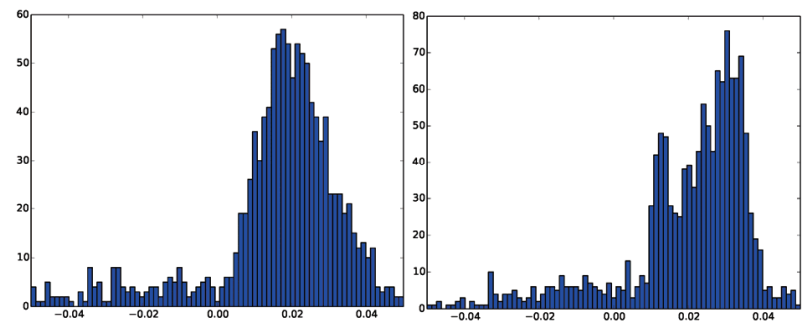

Figure 7. Histogram of deviations between $-5 \mathrm{~cm}$ and $+5 \mathrm{~cm}$ before adjustment for scanner 1 (left) and 2 (right).

To illustrate the deviations, Fig. 8 shows the residuals of equation (1), plotted along the trajectory (exemplarily for scanner 1). That is, for each point correspondence, the residual vector is determined (which is the normal vector $n_{i}$ times the distance to the surface), and plotted at the location of the mapping van when the point was measured. Note that the three graphs show the components of the residuals in $x, y$, and $z$, but do not directly indicate the required correction for the trajectory. For example, a point on a facade (with mainly horizontal normal vector) will lead to a zero value in the $z$ plot (lowest plot), although the corresponding equation (1) will not introduce a constraint in $z$. However, from Fig. 8, we can conclude that there is a shift in $y$ along the trajectory (middle plot), which is confirmed by the "green-to-red" pattern along the facades in Fig. 6. Also, in the lowest plot, we can see several point clusters in the $0.0 \mathrm{~m}$ to $0.1 \mathrm{~m}$ range, which correspond to the mainly red and orange points on the road surface in Fig. 6.

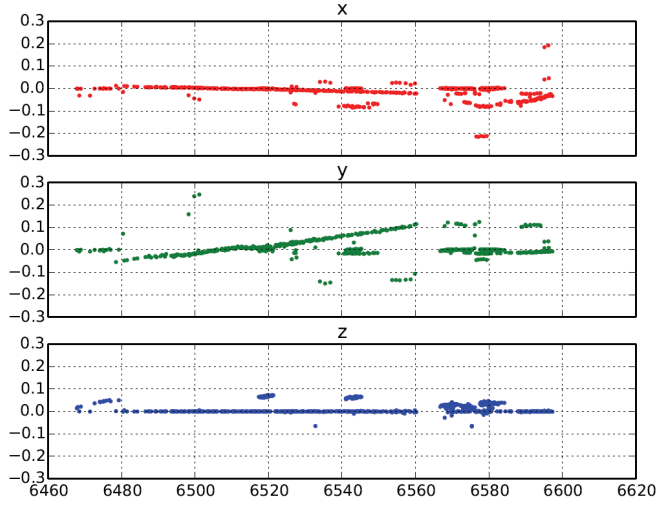

Figure 8 . Residual vectors along the trajectory from linear position $6470 \mathrm{~m}$ to position $6600 \mathrm{~m}$ before adjustment, for scanner 1. Components of the residual vector are split into $x, y$ and $z$ plots.

In our adjustment, described in section 4.2.3, we assume the following standard deviations: $2 \mathrm{~cm}, 10 \mathrm{~m}, 1 \mathrm{~cm}$ for equations (1), (2) and (3). This fixes the position only very weakly (equation 2), but introduces relatively strong constraints along the trajectory. The result of the adjustment is shown in Fig. 9. It can be seen that the result corresponds to the residuals plotted in Fig. 8. Also, it can be seen from the standard deviations (shown as curve thickness), that $y$ is well observed in contrast to $x$, which is due to the many points on the facade, which fix $y$ (across track), but not $x$ (along track). Also, the reference points on the road surface, which occur in several strips, lead to reduced standard deviations of $z$.

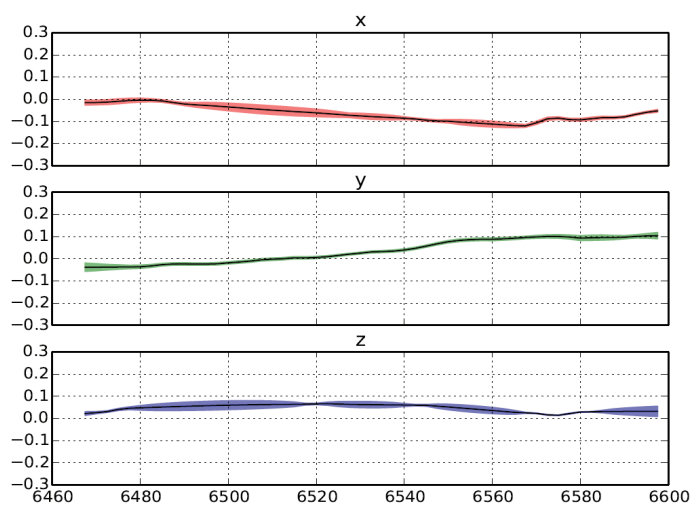

Figure 9. Adjustment result for the anchor points between linear position $6470 \mathrm{~m}$ and $6600 \mathrm{~m}$ with bounded variations in local neighbourhood (see Eq. 3). In each curve, the central line is the estimated value, where the band indicates the interval of one standard deviation.

The effect of equation (3) is noticeable when comparing Fig. 9 to Fig. 10. By applying equation (3) in the adjustment process, local variations between neighbouring anchor points are bounded, resulting in a small standard deviation (Fig. 9). In comparison, Fig. 10 shows the results of the adjustment without applying equation (3), which results in a large standard deviation noticeable by the wide band around the curves. In this figure, it becomes even more obvious that $y$ is well observed in contrast to $x$. 


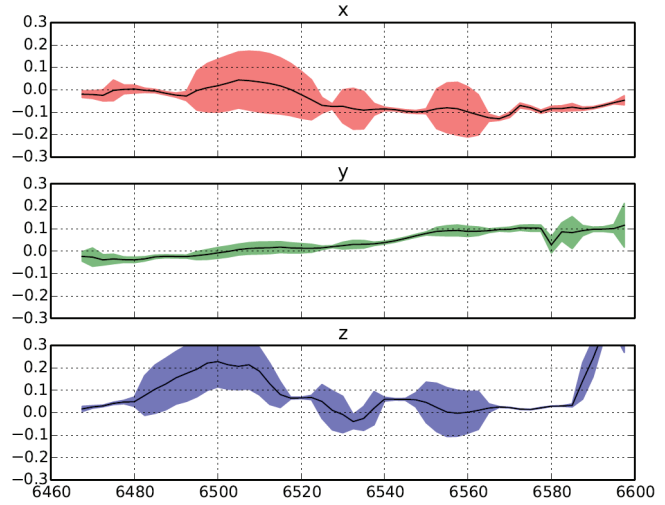

Figure 10. Adjustment result for the anchor points without bounded variations in local neighbourhood (compare to Fig. 9).

After applying the adjustment to the scan strips, the deviations shown in Fig. 11 are obtained. Outliers are shown in magenta. In both images, the rightmost reference points on the road show cyan points, which are due to a road renovation that occurred between the laser scanner and reference campaign.
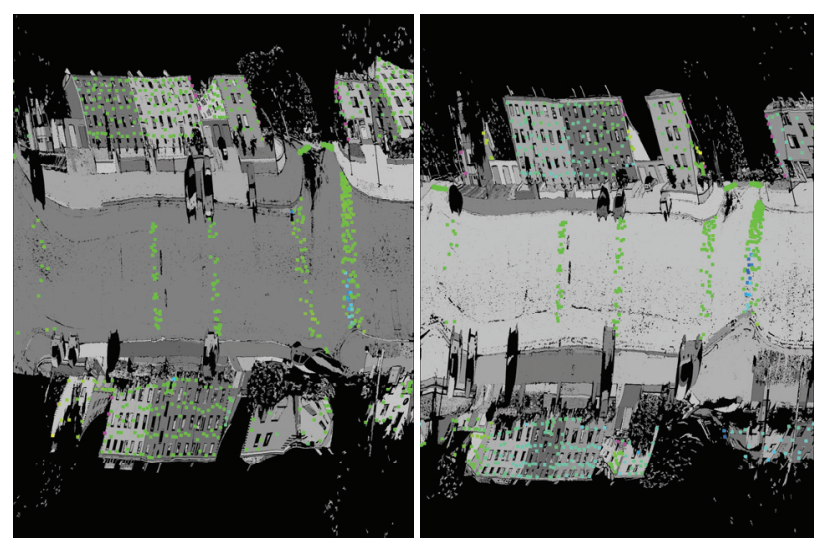

Figure 11. Colour coded deviations between reference and point cloud after adjustment of the trajectory for scanner 1 (left) and scanner 2 (right) with red $=+5 \mathrm{~cm}$, green $=0 \mathrm{~cm}$, blue $=-5 \mathrm{~cm}$, magenta: detected outliers.

From Fig. 12 (compare to Fig. 8), one can see that the residuals are mostly zero now for scanner 1 . However, scanner 2 (Fig. 11, right) shows an unexpected behaviour in the adjusted data. While for scanner 1, facades on both sides of the street appear to be green, they are cyan for scanner 2, which corresponds to contradicting requirements for the shift vector. We assume that this is due to some calibration error, which is to be investigated in the future.

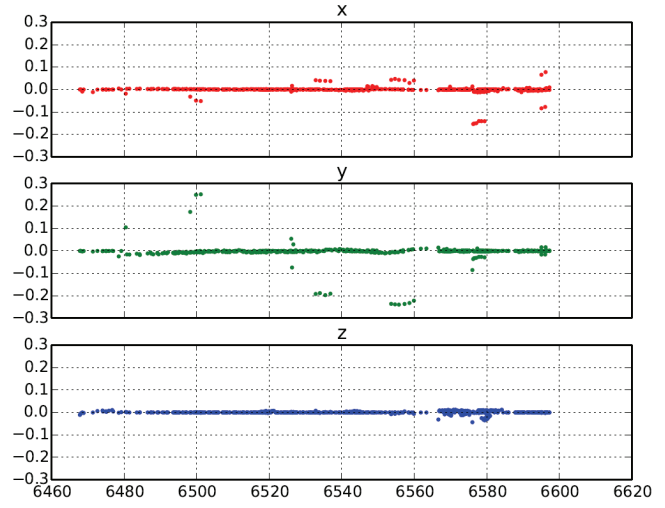

Figure 12. Determined shift at each reference point along trajectory after adjustment, exemplarily for scanner 1 .

The histogram of the deviations after adjustment is shown in Fig. 13 for scanner 1 (left) and scanner 2 (right). The peak of the deviations of scanner 1 is shifted to $0.0 \mathrm{~m}$ with a standard deviation of $6 \mathrm{~mm}$ which reflects the accuracy of the scanner. For scanner 2, we obtain two peaks, the minor peak being at $-2 \mathrm{~cm}$, which supports the finding in Fig. 11, right. The calculated mean value for scanner $1(2)$ is $0.2 \mathrm{~mm}(3.2 \mathrm{~mm})$ and the standard deviation is $5.7 \mathrm{~mm}(8.4 \mathrm{~mm})$.
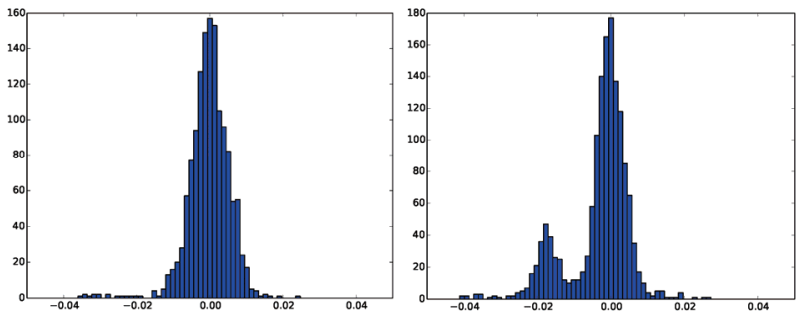

Figure 13. Histogram of deviations between $-5 \mathrm{~cm}$ and $+5 \mathrm{~cm}$ after adjustment for scanner 1 (left) and 2 (right).

Finally, the results for all data sets are presented in Fig. 14. In total, 32,951 $(33,341)$ observations were used for scanner 1 (2), in all epochs. The calculated mean value for all epochs for scanner 1 (2) is $1.1 \mathrm{~mm}(-5.3 \mathrm{~mm})$ and the standard deviation $7.2 \mathrm{~mm}(11.1 \mathrm{~mm})$. Again, the larger value for scanner 2 is due to the additional peak at $-2 \mathrm{~cm}$, which can clearly be seen in Fig. 14, right.
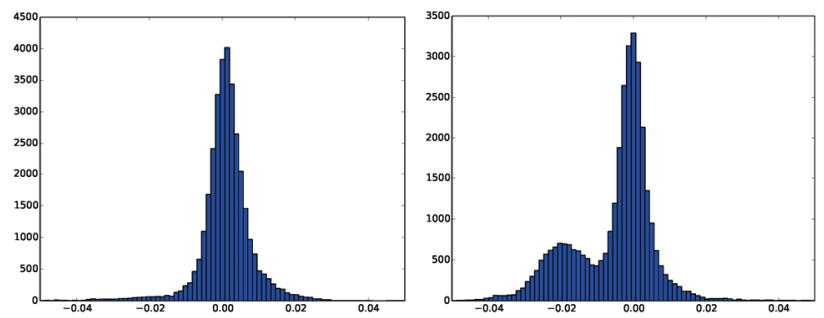

Figure 14. Histogram of deviations between $-5 \mathrm{~cm}$ and $+5 \mathrm{~cm}$ after adjustment for scanner 1 (left) and 2 (right), for 28 epochs. 


\subsection{Advantages and Drawbacks}

5.3.1 Advantages: The main advantage of our terrestrial reference data acquired by using a total station is the verified high accuracy for each point of the acquired reference data in comparison to other available data sources, such as cadastral data. Based on a geodetic network the accuracy of each stationing is well known and therefore, the accuracy of each reference point or object can be determined. Furthermore, the accuracy of such a test area is an order of magnitude better than the expected accuracy of the mobile mapping point clouds. Additionally, suitable objects can selectively be measured and exactly fit the needs for comparison with mobile mapping point clouds. During the selection process, especially stable objects can be chosen as reference objects, such as facades. Moreover, there is no need for installation or maintenance of targets, which could lead to problems in urban areas, e.g. permissions needed for installation of visible targets or even destruction by vandalism.

5.3.2 Drawbacks: On the other hand, the costly and time consuming acquisition of the reference data, especially the expenses for the geodetic network, must be mentioned as drawbacks. The data acquisition of the described reference test field with an extend of $300 \mathrm{~m} \times 300 \mathrm{~m}$ took about four days and two persons working, additionally, three days for set up, measurement and data processing for the geodetic network have to be planned. The expenses increase proportionally with the size of the captured reference test field. Therefore, the reference data may only be acquired on selected representative areas.

\section{CONCLUSION AND OUTLOOK}

\subsection{Conclusion}

In this work, we discussed a highly precise terrestrial test field with verifiable accuracy as reference data for mobile mapping point clouds in a characteristic urban area. The measurements of the test field are based on a geodetic network and include data of vertical planar facade elements for analysing the accuracy of the horizontal location, and street profiles for height accuracy analysis.

The accuracy analysis is based on a least squares strip adjustment using the deviation between the reference data and planar objects in the point clouds in the direction of their normal vectors. Before the adjustment, the standard deviation is in the range of $8 \mathrm{~cm}$, which can be reduced during the adjustment to $1.1 \mathrm{~mm}$ for scanner 1 and $-5.3 \mathrm{~mm}$ for scanner 2 with a standard deviation of $7.2 \mathrm{~mm}$ and $11.1 \mathrm{~mm}$, respectively. Differences between the reference data set and the point clouds, e.g. from road construction, are detected and removed as outliers during the adjustment.

\subsection{Outlook}

Further investigations on precision and accuracy of mobile mapping data can be made using pole like objects. In the reference data, a total of 30 pole-like objects, such as poles of street lights, traffic signs or trees trunks, were captured in scanning mode from opposite viewpoints if possible. The surface of these objects is acquired with great detail using the scanning mode of the total station, these objects may be used for more detailed analysis of the mobile mapping point clouds.
Additionally, the reference data or selected points or objects thereof may be used as ground control points for a point cloud adjustment of the mobile mapping point clouds from different epochs. For scanner 2, there are more investigations needed, regarding the additional peak at $-2 \mathrm{~cm}$ shown in Fig. 14 . Furthermore, the development of a simultaneous calibration procedure can be considered.

\section{REFERENCES}

Applanix Corp., 2012. POSLV Specifications. http://www.applanix.com (15 Feb. 2016).

AXIO-NET GmbH, 2016. GNSS precise real-time service. http://www.axio-net.eu (15 Feb. 2016).

Barber, D., Mills, J., Smith-Voysey, S., 2008. Geometric validation of a ground-based mobile laser scanning system. In: ISPRS Journal of Photogrammetry and Remote Sensing Vol. 63 (1), pp. 128-141, Theme Issue: Terrestrial Laser Scanning.

Graefe, G., Caspary, W., Heister, H., Klemm, J., Sever, M., 2001. The road data acquisition system MoSES - determination and accuracy of trajectory data gained with the Applanix POS/LV. In: 3rd International Symposium on Mobile Mapping Technology. Cairo, Egypt.

Haala, N., Peter, M., Kremer, J., Hunter, G., 2008. Mobile Lidar Mapping for 3D Point Cloud Collection in Urban Areas A Performance Test. In: The International Archives of the Photogrammetry, Remote Sensing and Spatial Information Sciences Vol. 37, pp. 1119-1127.

Kremer, J., Hunter, G., 2007. Performance of the Streetmapper Mobile LIDAR Mapping System in Real WorldProjects. In: Photogrammetric Week 2007. Wichmann, pp. 215-225.

LGLN Landesvermessung und Geobasisinformation Niedersachsen, 2016. Geodatenportal Niedersachsen. http://www.geodaten.niedersachsen.de (15 Feb 2016).

Leica, 2016. Data sheet Leica Nova MS 50 and Leica TS 30. http://www.leica-geosystems.de (15 Feb 2016).

Puente, I., Gonzalez-Jorge, H., Riveiro, B., Arias, P., 2012. Accuracy verification of the Lynx Mobile Mapper system. In: Optics \& Laser Technology, Vol. 45, pp. 578-586.

Riegl LMS GmbH, 2012. Data sheet Mobile Mapping System RIEGL VMX-250. www.riegl.com (15 Feb 2016).

Toschi, I., Rodríguez-Gonzálvez, P., Remondino, F., Minto, S., Orlandini, S., Fuller, A., 2015. Accuracy Evaluation of a Mobile Mapping System with Advanced Statistical Methods. In: ISPRS - International Archives of the Photogrammetry, Remote Sensing and Spatial Information Sciences, Volume XL5/W4, 2015, pp.245-253. 